\title{
Coxsackie A Virus
}

National Cancer Institute

\section{Source}

National Cancer Institute. Coxsackie A Virus. NCI Thesaurus. Code C112242.

A species of non-enveloped, spherical viruses with a capsid with $\mathrm{T}=3$ icosahedral symmetry in the Picornaviridae family and Enterovirus genus. The genome is composed of single-stranded, positive-sense RNA. Coxsackie A viruses are found in humans and cause hand, foot, and mouth disease, acute hemorrhagic conjunctivitis and herpangina via fecal-oral contamination. 